Fukushima J. Med. Sci.,

Vol. 48, No. 2, 2002

[Regular Papers]

\title{
USEFULNESS OF THE TACTILE SENSOR FOR ESTIMATING THE DEGREE OF LIVER FIBROSIS AND THE DNA SYNTHESIS ACTIVITY OF REMNANT LIVER CELLS AFTER PARTIAL HEPATECTOMY
}

\author{
YUICHI HATAKEYAMA ${ }^{1)}$, TOSHIYUKI ONO ${ }^{1)}$, NAOKI SATO'), \\ HIROSHI SAKUMA ${ }^{1)}$, YOSHIHISA KOYAMA ${ }^{1)}$, NORIO INOUE ${ }^{1)}$, \\ SEIICHI TAKENOSHITA ${ }^{1)}$ and SADAO OMATA ${ }^{2)}$ \\ 1) Department of Surgery II, Fukushima Medical University, ${ }^{2)}$ Department of Electrical and Electronic \\ Engineering, College of Engineering, Nihon University
}

(Received August 22, 2002, accepted October 17, 2002)

\begin{abstract}
Background/Purpose. Most patients with hepatocellular carcinoma in Japan also have liver cirrhosis. Therefore, reliable information about liver function is essential before undertaking liver resection. Occasionally preoperative estimates and intraoperative findings are disparate. This study used rats with liver fibrosis and a new tactile sensor to determine whether quantification of liver hardness intraoperatively can be used to validate the preoperative assessment of liver function prior to hepatectomy.

Methods. Liver fibrosis was induced by administration of thioacetamide to rats. Laparotomy was performed. In some rats, blood samples were taken for examination, and liver stiffness and tactile values were measured by a new tactile sensor. A biopsy specimen from each removed liver was taken, and the hepatic fibrosis index was measured by computed color image analysis after Azan blue staining. The other rats underwent partial hepatectomy, and the bromodeoxyuridine labeling index was calculated in them. Correlations between stiffness, tactile values, and other data were calculated.

Results. A strong correlation existed between stiffness and both the hepatic fibrosis index and the bromodeoxyuridine labeling index, and between tactile values and both the hepatic fibrosis index and the bromodeoxyuridine labeling index.

Conclusions. Liver hardness proved to be a function of hepatic fibrosis. The tactile sensor proved to be an easy and reliable way to evaluate the hepatic fibrosis index. Quantification of liver hardness by tactile sensor predicted liver regenerative activity.
\end{abstract}

Key words : liver hardness, liver cirrhosis, chronic hepatitis, liver resection, liver

畠山優一，小野俊之，佐藤尚紀，佐久間浩，小山善久，井上典夫，竹之下誠一，尾股定夫

Reprint requests to: Yuichi Hatakeyama, Department of Surgery II, Fukushima Medical

University School of Medicine, Fukushima City 960-1295, Japan. 
function

\section{INTRODUCTION}

Most cases of hepatocellular carcinoma in Japan are associated with liver cirrhosis $^{1)}$. Therefore, reliable information on liver function is needed before undertaking hepatectomy. A number of methods for assessing liver function, especially hepatic reserve, have been developed ${ }^{2-4)}$. However, we occasionally encounter a discrepancy between the preoperative estimation and the intraoperative findings. If it is possible to utilize intraoperative findings to refine preoperative estimates of hepatic function, these data can be used to finalize decisions regarding hepatic resection.

In this study, we measured hardness of the liver as determined by a new tactile sensor and correlated the results with the DNA synthesis activity of remnant liver cells after partial hepatectomy.

\section{METHODS}

Animals. Forty-four 5 -week-old Wistar rats were housed in individual cages with a 12 -hr light-dark cycle at $22 \pm 1.5^{\circ} \mathrm{C}$. All animals were maintained on water and rat chow (CLEA Japan, Inc., Tokyo, Japan) ad libitum until the day before surgery.

Induction of liver fibrosis. Liver fibrosis (chronic hepatitis and liver cirrhosis) was induced by intraperitoneal administration of thioacetamide (TAA) (200 mg/kg, 3 times/wk) for 12,10 , or 8 weeks in 34 rats. Ten healthy control rats were given saline on the same schedule.

Surgery. Laparotomy was performed all under pentobarbital $(30 \mathrm{mg} / \mathrm{kg})$ anesthesia 2 weeks after the last dose of TAA. Liver hardness was measured as follows. In 16 TAA-treated rats and 7 healthy control rats, blood samples were taken from inferior vena cava (IVC) and the prothrombin time (PT), hepaplastin test (HPT), glutamic pyruvic transaminase (GPT), alkaline phosphatase (ALP), total serum bilirubin (TB), and procollagen III peptide (PIIIP) concentrations were measured. In the other 18 TAA-treated rats and 3 healthy control rats, partial (70\%) hepatectomy was performed, and hepatic regenerative activity was determined by measuring bromodeoxyuridine (BrdU) incorporation into DNA $24 \mathrm{hr}$ after hepatectomy. In all rats, a biopsy specimen from the removed liver was taken and placed in $10 \%$ buffered formalin for histologic examination.

Quantification of liver hardness. A new measuring system was developed to estimate the degree of liver hardness. The instrument consists of a displacement transducer to measure a stress-strain curve of a soft tissue and a piezoelectric tactile sensor to obtain the acoustic characteristics of an object. Stiffness values of 
soft tissues were calculated from the stress-strain curves obtained by the degree of depression and the amount of counter weight by using the counter balance method. Tactile values were obtained in the following manner. In general, when a vibrating rod touches an object, its resonance frequency shifts as a function of the physical properties of the material. The new tactile sensor system is based on the contact compliance method ${ }^{5,6)}$. The sensor probe consists of a piezoelectric element and a vibration pickup element (Fig. 1). When an alternating voltage is applied across its electrode, the feedback circuit system oscillates at the resonance frequency of the piezoelectric element. A 3-mm diameter nylon hemisphere is attached to the free end of the piezoelectric element. When this end is pressed against an object surface, the resonance frequency varies according to the acoustic impedance of the object. Object hardness is measured by the tactile sensor, and the output, termed tactile values, are represented as the change in frequency, $\Delta f=f_{x}-f_{0}$, which is the difference between the resonance frequency of the points to which the sensor is applied, $f_{x}$, and the non-contact resonance frequency of the tactile sensor, $f_{0}$.

After laparotomy, we measured liver hardness at 3 points on each liver using the parameters, stiffness and tactile value, and the results average.

Histopathologic study. Representative blocks from each excised liver specimen were prepared. After routine paraffin-wax processing, sections were stained with hematoxylin and eosin, and the degree of liver damage was assessed under light microscopy. The hepatic fibrosis index was then calculated to quantify the degree of liver fibrosis in the following manner. Azan-stained sections were prepared, and
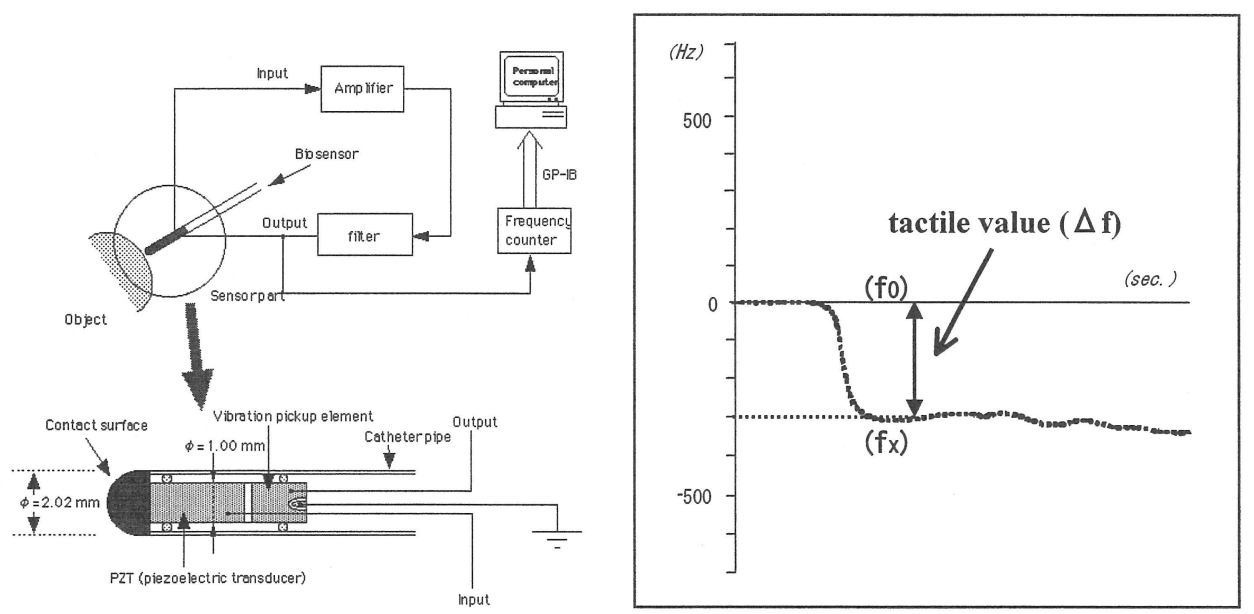

Fig 1. Schema showing the tactile sensor system. The system based on the contact compliance method. The sensor probe consists of a piezoelectric element and a cibration sonsor. A 3-mm diameter nylon hemisphere is attached to the free end of the piezoelectric element. Object hardness is measured bu the tactile sensor, (the tactile values), and is expressed as the change in frequency, $\Delta f=f_{x}-f_{0}$, which is the differnce between the resonance frequncy of the points to which the sensor is applied $\left(f_{x}\right)$, and the non-contact resonance frequency of the tactile sensor $\left(f_{0}\right)$. 
fibrous tissue was stained blue differentially. The percent of fibrosis, the blue area, was determined by computed color image analysis (Color Image Processor SPICCA-II, Nippon Avionics, Co., Tokyo, Japan). For each rat, the area of fibrous tissue was measured in five randomly selected fields, and the average was expressed as fibrosis per unit area of liver tissue (hepatic fibrosis index, HFI) ${ }^{7)}$.

Liver regenerative activity. Eighteen TAA-treated rats and 3 healthy control rats were killed by peritoneal pentobarbital overdose $24 \mathrm{hr}$ after partial hepatectomy. BrdU (50 mg/kg) was injected intraperitoneally $1 \mathrm{hr}$ before the rats were killed. The remnant livers were removed from the rats, and immunohistologic staining using anti-BrdU monoclonal antibody was performed. Incorporation of BrdU into hepatic DNA was employed as a marker of DNA synthesis and liver regenerative activity, termed the BrdU labeling index (BLI).

Statistics. Results are expressed as the mean \pm S.D. Statistical analysis was performed using Spearman's regression analysis where appropriate. Significance was accorded to $\mathrm{P}$ values less than 0.05 .

This study was approved by the Fukushima Medical University Animal Ethics Review Committee.

\section{RESULTS}

Histopathologic study. Healthy control rats (control) had an HFI of $0.841 \pm$ $0.601 \%$ and normal liver histology on light microscopy. On the other hand, rats treated with TAA exhibited varying degrees of liver fibrosis, showing more extensive hepatic fibrosis than any healthy rats. Rats treated with TAA for 8 weeks (TAA8) had an HFI of $3.762 \pm 1.238 \%$ and the appearance of chronic hepatitis on histology. Rats treated with TAA for 10 or 12 weeks (TAA10/12) had an HFI of $10.693 \pm 3.676 \%$ and the appearance of macronodular cirrhosis (Fig. 2). The mean HFI was different between groups $(p<0.0001)$.

Liver hardness. Control rats had stiffness values less than $8.7 \mathrm{~g} / \mathrm{cm}(6.049 \pm$ $1.230 \mathrm{~g} / \mathrm{cm})$ and tactile values less than $-2200(-2840.395 \pm 311.795)$ : TAA8 rats had stiffness values of $7.785 \pm 0.957 \mathrm{~g} / \mathrm{cm}$ and tactile values of $-2636.606 \pm 309.694$; and TAA10/12 rats had stiffness values of $10.004 \pm 1.029 \mathrm{~g} / \mathrm{cm}$ and tactile values of $-2239.803 \pm 208.946$. The mean stiffness value was different between each group $(p<0.0001$ between TAA10/12 and TAA 8 or control, $p=0.0005$ between TAA 8 and control). The mean tactile value was higher in TAA10/12 and TAA8 than control $(p<0.0001)$.

Liver hardness and laboratory data. Table 1 shows the correlations between stiffness, tactile values and laboratory data. There was a correlation between stiffness values and PT, HPT, ALP, PIIIP, and between tactile values and HPT, ALP, PIIIP.

Liver hardness and HFI (Fig. 3). The stiffness values and the HFI showed a positive correlation $(r=0.758, p<0.0001)$, as did the correlation between tactile 


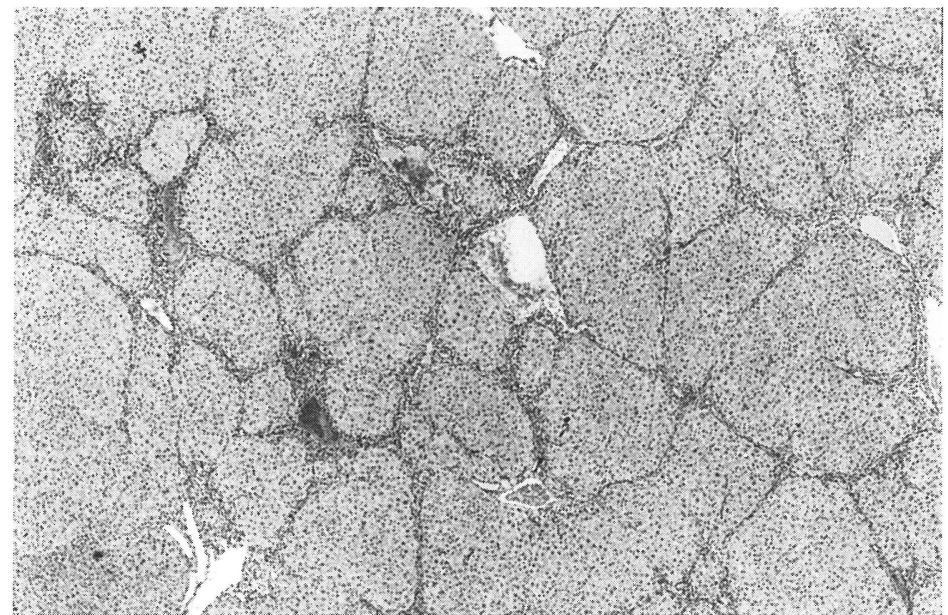

Fig 2. Photomicrograph of rat liver exposed to thioacetamide for 10 weeks showsmacronodular cirrhosis. (hematoxylin-eosin, $10 \times$ )

Table 1. Relationship between stiffness, tactile values, and laboratory data.

\begin{tabular}{c|c|c}
\hline & Stiffness value & Tactile value \\
\hline PT & $r=0.441, p=0.0349$ & $r=0.203, p=0.3535$ \\
\hline HPT & $r=-0.785, p<0.001$ & $r=-0.723, p=0.0003$ \\
\hline GPT & $r=0.577, p=0.0040$ & $r=0.210, p=0.3361$ \\
\hline ALP & $r=0.754, p<0.0001$ & $r=0.543, p=0.0074$ \\
\hline T-Bil & $r=0.204, p=0.3509$ & $r=-0.004, p=0.9873$ \\
\hline PIIIP & $r=0.832, p<0.001$ & $r=0.739, p=0.0001$ \\
\hline
\end{tabular}

$\square$ significant $\square$ not significant

PT: prothrombin time; HPT: hepaplastin test; GPT : glutamic pyruvic transaminase; ALP: alkaline phosphatase; TB: total serum bilirubim; PIIIP: procollagen III pepride.

values and HFI ( $r=0.651, p=0.008)$.

Liver hardness and Liver regenerative activity (Fig. 4). Control rats had a BLI of $48.367 \pm 5.954 \%$; TAA 8 rats had a BLI of $40.483 \pm 5.161 \%$; and TAA $10 / 12$ rats had a BLI of $24.983 \pm 5.799 \%$. The mean BLI was lower in TAA10/12 than either both TAA8 or control rats $(p<0.0001)$. Stiffness showed a negative correlation with BLI $(r=-0.811, p=0.0001)$. The correlation between tactile values and BLI also was negative $(r=-0.728, p=0.0002)$. 
A

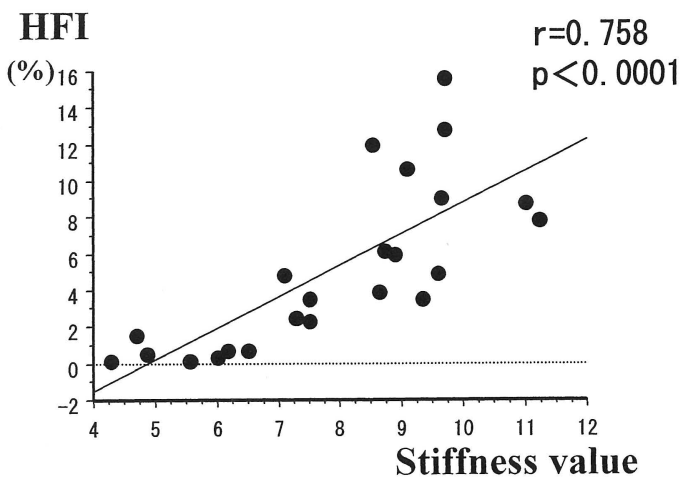

$(n=23)$

$\mathrm{B}$

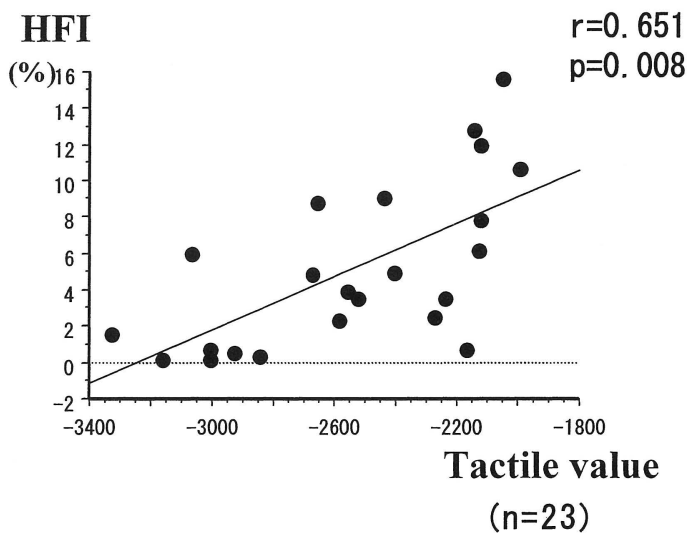

Fig 3. Correlation between liver hardness and the hepatic fibrosis index (HFI). A. A positive correlation ecisted between the stiffness value and the HFI $(r=0.758, p<$ 0.0001). B. A positive correlation existed between the tractile velue and the HFI $(r=0.651, p=0.008)$

\section{DISCUSSION}

The approximately $70 \%$ of Japanese $\mathrm{HCC}$ patients are positive for $\mathrm{HCV}$ antibody $(\mathrm{HCV}+)^{1}$. Therefore, if $\mathrm{HCV}+$ patients are followed regularly, $\mathrm{HCC}$ should be detected at an early stage. Unfortunately, however, most patients are not diagnosed until they become symptomatic. By then, liver function is often abnormal, and the cancer is at an advanced stage, precluding the use of less invasive therapies such as percutaneous ethanol injection therapy (PEIT) or microwave coagulation therapy $(\mathrm{MCT})^{8,9,10)}$. Surgical treatmet such as hepatectomy are indicated in these patients. Approximately $70 \%$ of HCC patients in Japan have liver cirrhosis and some degree of liver dysfunction ${ }^{11)}$. Therefore, reliable assessment of liver function is essential in planning surgery.

Estimates of Hepatic functional reserve have been used as the primary determi- 
A

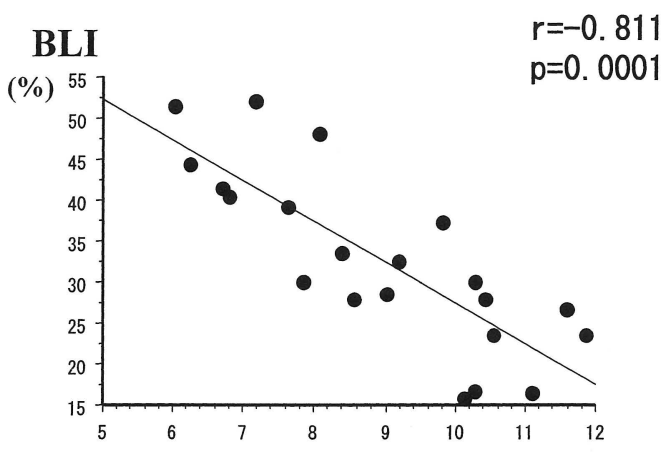

Stiffness value

$(n=21)$

B

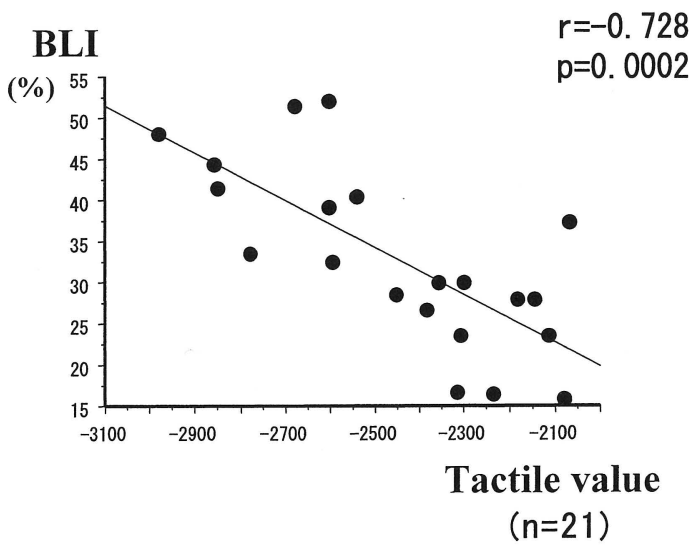

Fig 4. Correlation between liver hardness and bromodeoxyuridine labeling index (BLI). A. A negative correlation existed between stiffness value and the BLI $(r=-0.811, p=0.0001)$. B. A negative correlation existed between the tactile value and the BLI $(r=-0.728, p=0.0002)$.

nant of a patient's capacity to undergo hepatectomy. Mizumoto et al. ${ }^{2)}$ analyzed various parameters of hepatic function and created an equation to estimate the risk of liver resection in patients with HCC who have liver cirrhosis using Alb, GOT, TBil, Ch-E, PT, K ICG, OGTT, HPT, and LCAT. Yamanaka et al. ${ }^{3)}$ performed multivariate analysis on parameters of hepatic function and estimated the risk of hepatectomy based on the resection ratio, age, ICG R15, and ICG Rmax. Mori and

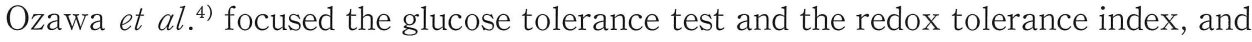
reported their clinical utility. These methods have been used in our hospital and have been helpful in selecting the type of operation. However, we occasionally observe a discrepancy between pre- and intra-operative liver findings. If it is possible to utilize intraoperative findings as the final check on liver function, it is conceivable that the capacity of the cirrhotic liver to tolerate hepatectomy could be determined more accurately. 
Initially we focused on the degree of liver fibrosis to estimate the capacity of the cirrhotic liver to tolerate hepatectomy, and performed an experiment using rats with liver cirrhosis. We found that the liver fibrosis index calculated by the Azanstaining method and computed color image analysis predicted liver regenerative activity $^{7}$. However, the limitation to this method is the need to obtain a large block of liver by biopsy.

We hypothesized that liver hardness would correlate with the degree of liver fibrosis. Some authors have tried to measuresized liver hardness and use it as an index of liver function during hepatic surgery ${ }^{12,13)}$. However their methods were invasive. Omata ${ }^{5,6}$ developed a new tactile sensor that enabled us to quantify the hardness of tissues and organs. Only the sensor tip touches the tissue, so hardness is measured very easily and rapidly. Before this study, we used the tactile sensor to study the relationship between the hardness of breast cancer and the histopathology and found that the sensor predicted the histopathologic types of breast cancer reliably, and that it was safe and practical for human use $\mathrm{e}^{14)}$.

In this study, we treated rats with thioacetamide as a model of liver cirrhosis. It has been reported that thioacetamide-induced liver cirrhosis is closer to human cirrhosis than the carbon tetrachloride model based on histology and hemodynamics ${ }^{15-17)}$. The hepatic fibrosis index has been shown to correlate with the hydroxyproline content of cirrhotic liver ${ }^{18}$. We used the BLI as the indicator of liver regenerative activity because $\mathrm{BrdU}$ is an analogue of thymidine and participates in DNA replication. BrdU is taken up by hepatocytes during the S-phase of mitosis and is incorporated into DNA at that point ${ }^{19)}$. Since liver regenerative activity is determined by proliferation of remnant liver cells ${ }^{20)}$, measuring the amount of BrdU incorporated into liver serves as an index for estimating liver regenerative potential.

Stiffness and tactile values were measured as parameters of liver hardness. There was strong correlation between the two and each showed similar correlations with the liver fibrosis index and liver regenerative activity. Therefore, it seems likely that the use of tactile values obtained by the new tactile sensor alone is adequate to estimate the liver fibrosis index and liver regenerative activity without needing to calculate stiffness.

In conclusion, we were able to quantify liver hardness easily and rapidly by using a new tactile sensor, and the quantified tactile value predicted the degree of fibrosis and regenerative activity after liver resection in rats with cirrhosis. We currently are working to develop this tactile sensor for clinical use.

\section{REFERENCES}

1. Arii S, Okamoto E, Imamura M, Registries in Japan: current status of hepatocellular carcinoma in Japan. Liver Cancer Study Group of Japan. Seminars in Surgical Oncology, 12, 204-211, 1996. 
2. Mizumoto R, Noguchi T, Selection of treatment for hepatocellular carcinoma with preoperative estimation of hepatic functional reserve. Shokakibyo Seminar (Gastroenterological Disease Seminar), 26, 6-18, 1987. (in Japanese)

3. Yamanaka N, Okamoto E, Kuwata K, Tanaka N, A multiple regression equation for prediction of posthepatectomy liver failure. Ann Surg, 200, 658-663, 1984.

4. Mori K, Ozawa K, Yamamoto Y, Response of hepatic mitochondrial redox state to oral glucose load-redox tolerance test as a new predictor of surgical risk in hepatectomy. Ann Surg, 211, 438-446, 1990.

5. Omata S, Measurement of the hardness of a soft material with a piezoelectric vibrometer and their analysis. Jpn J Med Electron Biol Eng, 28, 1-8, 1990.

6. Omata S, New tactile sensor like the human hand and its applications. Sensors and Actuators A, 35, 9-15, 1992.

7. Hatakeyama Y, Ono T, Abe R, The grade of hepatic fibrosis as a predictor of regeneration activity after partial hepatectomy to liver cirrhosis in the rat. Nihon Shokakigeka Gakkai Zasshi (Jpn J Gastroenterol Surg), 29, 691-698, 1996. (in Japanese)

8. Sasaki Y, Imaoka S, Nakano H, Ishikawa O, Ohigashi H, Yasuda T, Nakamori S, Kameyama M, Hiratsuka M, Kabuto T, Furukawa H, Kasugai H, Inoue A, Fujita M, Inoue E, Indications for hepatectomy for hepatocellular carcinoma - what stage of the disease is the best indication for surgery?. J Hepatobiliary Pancreat Surg, 5, 14-17, 1998.

9. Tabuse K, Basic knowledge of a microwave tissue coagulator and its clinical applications. J Hepatobiliary Pancreat Surg, 5, 165-172, 1998.

10. Nagasue N, Liver resection for hepatocellular carcinoma : indications, techniques, complications, and prognostic factors. J Hepatobiliary Pancreat Surg, 5, 7-13, 1998

11. The liver cancer study group of Japan, Primary liver cancer in Japan. Clinicopathologic features and results of surgical treatment. Ann Surg, 211, 277-287, 1990.

12. Nishizaki T, Matsumata T, Kamakura T, Adachi E, Sugimachi K, Significance of intraoperative measurement of liver consistency prior to hepatic resection. HepatoGastroenterol, 42, 5-8, 1995.

13. Yamanaka N, Okamoto E, Toyosaka A, Ohashi S, and Tanaka N, Consistency of human liver. J Surg Res, 39, 192-198, 1985.

14. Hara K, Motooka N, Omata S, Mabuchi T, Kimijima I, Abe R, Development of mammography instrument using a new tactile sensor capable of sensing stiffness of tissue. Technical digest of the 14th sensor symposium, 14, 101-104, 1996

15. Tamayo MP, Is cirrhosis of the liver experimentally produced by $\mathrm{CCl} 4$ an adequate model of human cirrhosis? Hepatology, 3, 112-120, 1983.

16. Zimmermann T, Muller A, Machnik G, Franke H, Schubert H, Dargel R, Biochemical and morphological studies on production and regression of experimental liver cirrhosis induced by thioacetamide in Uje WIST rats. Z Versuchctierkd, 3, 165-180, 1987.

17. Dashti H, Jeppsson B, Haggerstand I, Hultberg B, Srinivas U, Abdulla M, Bengmark S, Thioacetamide- and carbon tetrachloride-induced liver cirrhosis. Eur Surg Res, 21, 8391, 1989

18. Jimenez W, Pares A, Caballeria J, Heredia D, Brugera M, Torres M, Rojkind M, Rodes J, Measurement of fibrosis in needle liver biopsies: Evaluation of a colorimetric method. Hepatology, 5, 815-818, 1985.

19. Gratzner HG, Monoclonal antibody to 5-bromo- and -5-indodeoxy uridine: A new reagent for detection of DNA replication. Science, 218, 474-475, 1982.

20. Luk GD, Essential role of polyamine metabolism in hepatic regeneration. Gastroenterology, 90, 1261-1267, 1986. 